\title{
Advance Impulse Noise Filtering using Double Threshold with Edge Preservation
}

\author{
Priyanshi Shrivastava \\ LNCTS Bhopal
}

\author{
Soni Changlani \\ LNCTS Bhopal
}

\begin{abstract}
Impulse noise detection and removal is very important process in image processing. Here random valued impulse noise (RVIN) is considered because it does not affect the gray values of image pixels uniformly. This paper have proposed an recursive double median median filter based noise removal method using double threshold which filter the impulse noise in two stages, first one is noise detection and second is noise removal. The mathematical calculation and analysis shows that proposed method provides better visual quality and higher PSNR as compared to existing denoising methods even when noise density is very high.
\end{abstract}

\section{Keywords}

SD-ROM, impulse noise, median filter, PSNR, MSE.

\section{INTRODUCTION}

During the transmission and acquisition, pixels gray values are affected by different type of noise present in medium. These noise can be Gaussian type or impulse noise[6]. The most common type of noise which affects the image in the form of black and white dots is impulse noise. Impulse noise is of two types

\section{1). Fixed value impulse noise and \\ 2). Random valued impulse noise.}

Fixed valued impulse noise is noise which can have values either 0 or 255 ( 0 for black and 255 for white). On the other hand random valued impulse noise can have 0,255 or any value between 0 and 255. So the different types of detection and removal algorithms are requiring for different types of noise. Filters are better option for image de-noising. There are so many filters already available for image de-noising. The mean filter or average is very simple and easy filter for image noise removal but while removing the noise, mean filter affects fine details of image and causes blurring into the image. Also the nature of impulse noise is non-linear so the non-linear filter will work most efficiently then mean filter.

Median filter also knows as simple median filter (SMF)[5]. It is very common used non-linear filter. The main concept of median filter is sorting. It sorts the all elements of the selected filtering window of size $n x n$, where $n$ is odd number. Then it chooses the middle element of the sorted seuence and then replaces this median element with the central noisy pixel. After replacing nisy pixel, that window will slides to the next pixel and repeat the process. This filter produces better results and quality than average and other linear filters. But it has one disadvantage that it also effects noise-free pixels. That is why some improvement has been made in median filter to get better results like first identify the noisy pixels and then replace only noisy pixels and other left unaltered. Weighted median filter (WMF)[4] is an improvement in the median filter. Some other filters are centre weighted median filter (CWM)[11][12], Adaptive median filter (AMF), Adaptive centre weighted median filter (ACWM)[4], Switching median filter (SMF)[3], progressive switching median filter (PSMF)
[9]. The combined median and other filter also use to improve the performance of median filter like median filter combine with average filtering and median filter with high pass filter.

\section{NOISE MODEL}

Impulse noise is very common type noise in digital images. [5] Impulse noise is completely independent and not correlated to the pixels and is randomly distributed over the entire image. Hence unlike Gaussian noise, for an corrupted image effected by impulse noise, all the image pixels are not noisy, only a number of image pixels are effected by impulse noise and the rest of pixels will be noise free. There are two types of impulse noise called as salt and pepper noise or fixed valued impulse noise and random valued impulse noise. In fixed type noise, the noisy pixels takes either salt value (gray level -225) or pepper value (gray level -0) and it distributed in form of black and white dots on the images. If $p$ represents the noise density then salt and pepper noise both will have a noise density of $\mathrm{p} / 2$.This can be mathematically represented in eq. (2.1).

$$
y_{i j}=\left\{\begin{array}{cc}
0 \text { or } 255 \text { with probability } p \\
x_{i j} \quad \text { with probability } 1-p
\end{array}\right.
$$

Where $y_{i j}$ represents the noisy image pixel, $\mathrm{p}$ is the total noise density of impulse noise and $x_{i j}$ is the uncorrupted image pixel. At times the salt noise and pepper noise may have different noise densities $p_{1}$ and $p_{2}$ thus the total noise density will be $\mathrm{p}=. p_{i}+p_{2}$

In case of random valued impulse noise, noise can take either 0,255 or any Gray level value from 0 to 225 . In this case also noise is randomly distributed over the entire image and probability of occurrence of any gray level value as noise will be same. We can mathematically represent random valued impulse noise as:

$y_{i j}=\left\{\begin{array}{lll}n_{i j} & \text { with probability } \quad p \\ x_{i j} & \text { with probability } 1-p\end{array}\right.$

Where $n_{i j}$ the gray level value of the noisy pixel is $x_{i j}$ is the uncorruptedimage pixel.

\section{DENOISING METHODS}

\subsection{Progressive Switching Median Filter (PSMF) [9]}

It is a progressive switching median filter(PSMF), proposed for the removal of Impulse Noise from Highly Corrupted Images. The filtering method is based on the following two main steps

(1) Switching stage: An impulse noise detection scheme is used before filtering, thus only a fraction of all the pixels will be subjected to filtering process.

(2) Progressive stage: Both the impulse detection and the noise filtering procedures are progressively applied through a number of iterations. The main advantage of this method is that some impulse pixels located in the middle of large noise 
blotches can also be properly detected and filtered, which results in better restoration, especially for the cases where the images are highly corrupted.

\subsection{Signal-Dependent Rank Ordered Mean Filter (SD-ROM) [10]}

It is an efficient nonlinear algorithm to suppress impulse noise from highly corrupted images while preserving imagedetails and features. The method is applicable to all impulse noise models, including fixed valued (equal height or salt and pepper) impulses and random valued (unequal height) impulses, covering the whole dynamic range. The technique achieves excellent tradeoff between the suppression of noise, and preserving the details and edges without undue increase in computational complexity.

\section{PROPOSED METHOD}

We have developed the simple algorithm in which we perform the noise detection \& noise removal process much efficiently. We use the smallest window size which preserves the fine details of image. The window of size $3 \times 3$ chooses for noise detection and noise removal. The window contains total 9 elements which are as follows:

Z1, Z2, Z3, Z4, Z5, Z6, Z7, Z8, Z9.

Our proposed method broadly divided in to two parts first is detection stage and second is noise elimination stage. In the proposed method, the random valued noise is added to the targeted image, now the noisy pixels are detected with reference to three different conditions. After the detection of noisy pixels they, are subjected to de-noising process according to noise density level. The complete de-noising process can be dividing in following number of steps:

Table 4.1: Filtering window of size 3X3

\begin{tabular}{|l|c|c|c|}
\hline & Column 1 & Column 2 & Column 3 \\
\hline Row 1 & $\mathrm{Z}_{1}$ & $\mathrm{Z}_{2}$ & $\mathrm{Z}_{3}$ \\
\hline Row 1 & $\mathrm{Z}_{4}$ & $\mathrm{Z}_{5}$ & $\mathrm{Z}_{6}$ \\
\hline Row 3 & $\mathrm{Z}_{7}$ & $\mathrm{Z}_{8}$ & $\mathrm{Z}_{9}$ \\
\hline
\end{tabular}

\section{Step-1}

First we will select filtering window of minimum size i.e., $3 X 3$. So there will be nine elements in this filtering window. But wenee to exclude central pixel for $3 \times 3$ windows to calculate maximum value and minimum value for remaining pixel. First of all we detect that pixel is corrupted by impulse noise or not. It has three possibilities;

1. If the central pixel value lies between maximum and minimum value of filtering window i.e.

$\operatorname{Max}[\mathrm{Zr}]>\mathrm{Zi}, \mathrm{j}>\operatorname{Min}[\mathrm{Zr}]$

then it considered as noise-free pixel.

2. If the central pixel value is greater than maximum value or smaller than minimum value of filtering window, i.e.

$\mathrm{Zi}, \mathrm{j}>\mathrm{Max}[\mathrm{Zr}]$ or $\mathrm{Zi}, \mathrm{j}<\mathrm{Min}[\mathrm{Zr}]$

then it is treated as noisy pixel.

3. If the central pixel value equals the minimum or the maximum valu of filtering window, then we need to identify whether it is an edge or a noisy pixel.to identify this, we divides the filtering window in four sub window, i.e. column subwindow, row subwindow and two diagonals subwindow. Then calculate sum of absolute difference between central pixel $\mathrm{Zi}, \mathrm{j}$ and its neighbors ( $\mathrm{d} 1, \mathrm{~d} 2, \mathrm{~d} 3, \mathrm{~d} 4)$.

$\mathrm{Zi}, \mathrm{j}=\mathrm{Max}[\mathrm{Zr}]$ or $\mathrm{Zi}, \mathrm{j}=\mathrm{Min}[\mathrm{Zr}]$

4. After calculating the sum we gor 4 different values i.e. d1, $\mathrm{d} 2, \mathrm{~d} 3, \mathrm{~d} 4$. Then we determine the minimum value among these 4 and its treated pivot point represented by. D. Now we compare it with predefined threshold(T). If $\mathrm{D}>\mathrm{T}$ (30) than its noisy pixel otherwise it is an edge, and pixel is noise free, after that we apply dual median method for the replacement of noisy pixel.

Step-2

The image obtained in the previous step is again denoised by calculating median value once more; the targeted pixel is replaced by this median value, hence a better denoised image is obtained with improved PSNR and reduced MSE.

\section{RESULTS AND SIMULATION}

Proposed de-noising technique is simulated using MATLAB 7.9 on Intel-core i-5 processor with 4 GB RAM. De-noising is performed on Lena image of size 256X256. We have analyzed the performance of existing and proposed methods by using performance parameters like PSNR (Peak Signal to Noise Ratio) and MSE (Mean Square Error). The simulation result shows that PSNR (Peak Signal to Noise Ratio) of de-noised image by this filter is better than other filters.

The PSNR (Peak Signal to Noise Ratio) is expressed as:

$$
P S N R=\operatorname{lOlog}_{10} \frac{(255)^{2}}{\mathrm{MSE}} .
$$

Where MSE (Mean Square Error) is

$$
M S E=\frac{\sum_{i=1}^{m} \sum_{j=1}^{n}\{Z(i, j)-A(i, j)\}^{2}}{m \times n}
$$

The results in the Table II clearly show that the PSNR of proposed method is better at high density random valued impulse noise. The results are compared with several standard median based filters like Median filter (SMF)[5], Centre weighted median filter (CWM)[14], Progressive switching median filter (PSM)[9],Signal dependent rank order median filter (SDROM)[8], Adaptive centre weighted median filter (ACWM)[14]. The fig 2(b),2(e),2(h), shows Lena image corrupted by $50 \%, 80 \%$ and $90 \%$ respectively and figure $2(\mathrm{c})$, 2(f), 2(i) show images De-noised by proposed method.

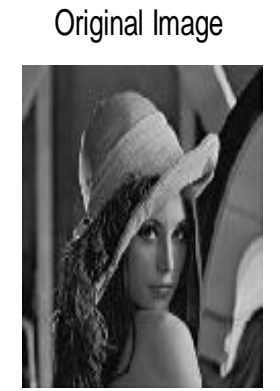

Fig 2(a)

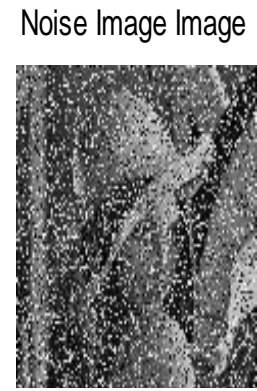

Fig 2(b)
Restored Image

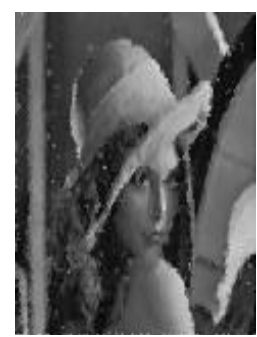

Fig 2(c)
Experimental Results of Proposed Method for Lena Image for $50 \%$ Noise density 
Original Image

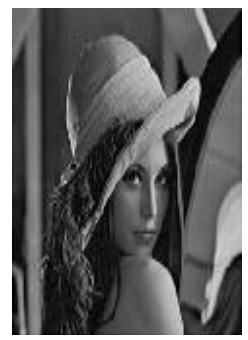

Fig 2(d)

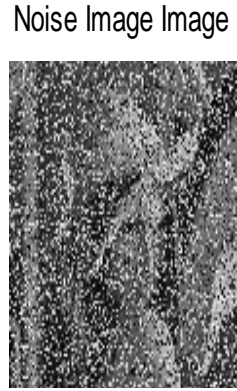

Fig 2(e)

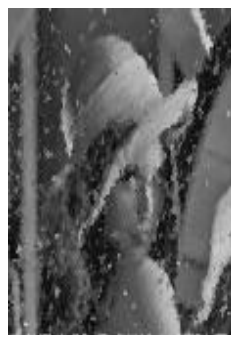

Fig 2(f)
Restored Image

Experimental Results of Proposed Method for Lena Image for $80 \%$ Noise density

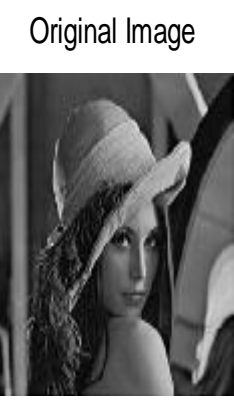

Fig 2(g)

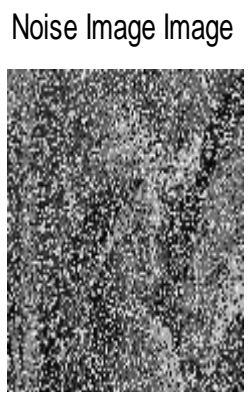

Fig 2(h)
Restored Image

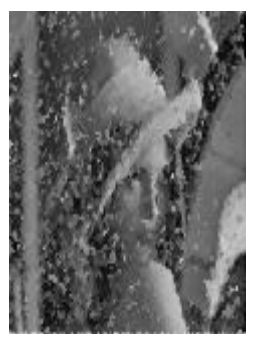

Fig 2(i)
Experimental Results of Proposed Method for Lena Image for $90 \%$ Noise density

Table 5.1 Comparision of PSNR Values of different Filters for Lena Image

\begin{tabular}{|c|c|c|c|c|c|}
\hline $\begin{array}{c}\text { De-noising } \\
\text { Methods }\end{array}$ & \multicolumn{5}{|c|}{ Noise density } \\
\hline & $50 \%$ & $60 \%$ & $70 \%$ & $80 \%$ & $90 \%$ \\
\hline MF & 14.7 & 12.3 & 10.9 & 8.3 & 6.8 \\
\hline CWM & 12.9 & 10.9 & 9.1 & 7.7 & 6.6 \\
\hline PSM & 19.5 & 15.3 & 11.0 & 8.9 & 6.5 \\
\hline SDROM & 14.3 & 11.8 & 9.6 & 7.9 & 6.6 \\
\hline IRF & 14.7 & 12.3 & 9.9 & 8.2 & 6.8 \\
\hline RACWM & 20.2 & 18.2 & 15.4 & 12.4 & 8.8 \\
\hline TSM & 12.6 & 10.5 & 8.6 & 7.2 & 6.2 \\
\hline LEAM & 23.1 & 21.5 & 19.6 & 17.8 & 14.9 \\
\hline $\begin{array}{c}\text { Proposed } \\
\text { Filter }\end{array}$ & 24.05 & 23.26 & 22.65 & 22.17 & 21.71 \\
\hline
\end{tabular}

Table 5.2Comparision of MSE Values of different Filters for Lena image

\begin{tabular}{|c|c|c|c|c|c|}
\hline \multirow{2}{*}{$\begin{array}{c}\text { De- } \\
\text { noising } \\
\text { Methods }\end{array}$} & \multicolumn{5}{|c|}{ Noise density } \\
\hline & $50 \%$ & $60 \%$ & $70 \%$ & $80 \%$ & $90 \%$ \\
\hline MF & 2203.33 & 3829.0 & 5285.43 & 9617.9 & 13585.6 \\
\hline CWM & 3334.88 & 5285.43 & 7999.82 & 11042.82 & 14225.9 \\
\hline PSM & 729.60 & 1919.00 & 5165.12 & 8376.84 & 14557.2 \\
\hline SDROM & 2104.16 & 3918.14 & 6967.55 & 10071.18 & 14557.2 \\
\hline IRF & 2415.90 & 4296.16 & 7129.85 & 10545.82 & 14225.9 \\
\hline RACWM & 620.98 & 984.19 & 1875.34 & 3741.80 & 8571.96 \\
\hline TSM & 3573.39 & 5795.36 & 8975.95 & 12390.25 & 15598.4 \\
\hline LEAM & 318.47 & 460.34 & 712.98 & 1079.14 & 2104.16 \\
\hline $\begin{array}{l}\text { Proposed } \\
\text { Filter }\end{array}$ & 258.87 & 301.57 & 346.08 & 392.77 & 448.21 \\
\hline
\end{tabular}

\section{CONLUSION}

The proposed filter has proved that it is very efficient for random valued impulse noise because practically noise is not uniform over the channel. We have used the concept of maximum and minimum threshold to detect both positive and negative noise. It produces very good PSNR (Peak Signal to Noise Ratio) and very small MSE (Mean Square Error) for highly corrupted images, especially for more than $50 \%$ noise density. This algorithm is simple and requires less number of calculations than other filters like CWM, TSM, SD-ROM, IMF etc. small size of filtering window gives advantage of preservation of fine details of image. Because of its less complexity of calculation, this filter will have great application in the field of image processing.. This method has the following advantages:

1) The median value is more accurate than other filters. Because we are not using simple median value but enhanced median value witch gives better results than simple median.

2)Double thresholding is used and calculated threshold values can change at each filtering window adaptively according to the percentage of noise in filtering window. Both thresholds are different for different noise density. Previous de-noising stretegies use either single thresholding or constant thresholding over the entire image irrespective of percentage of noise.

3) This algorithm require very less complex sorting method because very small amount of calculation are need for the selection of minimum values, maximum values and median values.

This method can have great application in the field of communication, because large amount of noise introduced during the transmission of data. This method can be implemented using parallel processing or pipelining. With the help of parallel processing we can speed up the process and reduce execution time. Because of its easy hardware 
implementation, this method can replace the existing enoising methods. It can be used in GPS system as well with some modification and can give better picture quality at high noisy environment.

\section{REFERENCE}

[1] Vikas Gupta and Abhishek Sharma "Image De-Noising by Enhanced Median Filtering for High Density Noisy Images", Proceedings of the Fourth International Conference on Signal and Image Processing 2012 (ICSIP 2012), Lecture Notes in Electrical Engineering 222, DOI: 10.1007/978-81-322-1000-9_55, _ Springer India 2013.

[2] Shanmugavadivu P \&EliahimJeevaraj P S, "Laplace Equation based Adaptive Median Filter for Highly Corrupted Images", International Conference on Computer Communication and Informatics (ICCCI 2012), 2012

[3] Bhabesh Deka, Dipranjan Baishnab "A Linear Prediction Based Switching Median Filter, for the Removal of Salt and Pepper Noise from Highly Corrupted Image", CISP2012, IEEE.

[4] WANG Chang-you, YANG Fu-ping, GONG Hui, “A new kind of adaptive weighted median filter algorithm"2010 International Conference on Computer Application and System Modeling (ICCASM 2010).

[5] J.Harikiran, B.Saichandana and B.Divakar "Impulse Noise Removal in Digital Images," International Journal of Ccmputer Applications vol. 10, no. 8, Nonember 2010 .

[6] Gonzalez R.C, Woods. R.E., Digital Image Processing, $3^{\text {rd }}$ edition, Pearson Prentice Hall, 2009.
[7] James C. Church, Yixin Chen, and Stephen V. Rice "A Spatial Median Filter for Noise Removal in Digital Images", Southeastcon, 2008

[8] T. Chen, K.-K. Ma, and L.-H. Chen,"Tri-state median filter for image denoising," IEEE Trans. Image Processing, vol. 8, no. 12, pp. 1834-1838, 1999.

[9] Z. Wang and D. Zhang, "Progressive switching median filter for the removal of impulse noise from highly corrupted images," IEEE Trans. on Circuits and Systems II: Analog and Digital Signal Processing, vol. 46, no. 1, pp. 78-80, 1999.

[10] E. Abreu and S. Mitra, "A signal-dependent rank ordered mean (SDROM) filter-a new approach for removal of impulses from highly corrupted images," in ICASSP, vol. 4, pp. 2371 -2374, 1995 IEEE.

[11] T. Song, M. Gabbouj, and Y. Neuvo, "Center weighted median filters: some properties and applications in image processing," Signal Processing, vol. 35, no. 3, pp. 213 229, 1994.

[12] S.-J. Ko and Y. Lee, "Center weighted median filters and theirapplications to image enhancement," IEEE Transactions on Circuits andSystems, vol. 38, no. 9, pp. 984-993, September 1991

[13] V. Jayaraj, D. Ebenezer, V. R. Vijayakumar, “A Noise Free Estimation Switching Median Filter for Detection and Removal of Impulse Noise in Images", European Journal of Scientific Research ISSN 1450-216X Vol.51 No.4 (2011), pp.563-581. 